\title{
La reforma del estatuto jurídico civil de los animales en el Derecho francés
}

\section{Loïs Lelanchon}

Abogado francés. Máster en Derecho Animal y Sociedad (UAB)

Experto consultor en Derecho Animal para entidades internacionales.

Recepción: Abril 2018

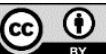

Aceptación: Junio 2018

Este trabajo forma parte del Proyecto de investigación MINECO DER2015-69314-P «Estatuto jurídico de los animales: origen, desarrollo y políticas» (2015-2019)

This work forms part of the MINECO investigation Project DER2015-69314-P «Legal status of animals: origin, development and policies» (2015-2019)

\section{Resumen}

Esta contribución se centra en la consideración de los animales en la legislación francesa, y más específicamente desde el ángulo de la modernización del estatuto legal de los animales en el derecho civil, operada en 2015, y que reconoce a los animales como seres sensibles. Esta contribución arroja algo de luz sobre los desafíos encontrados antes de la adopción de la reforma y las incertidumbres que aún persisten en cuanto a su valor legal y su impacto concreto para los animales.

Palabras clave: derecho civil, animales; derecho animal, propiedad; ser sensible, derecho penal, estatuto jurídico, Francia

\section{Abstract}

This contribution focuses on the consideration of animals in French law and more specifically from the angle of the modernisation of the legal status of animals in civil law, operated back in 2015, and that recognised animals as sentient beings. This contribution sheds some light on the challenges encountered prior to the adoption of the reform and the uncertainties that remain to this day with regards its legal value and the concrete impact for animals..

Keywords: civil law; animals; animal law; property; sentient being; criminal law; legal status; France. 


\section{SUMARIO}

\section{Introducción}

1. La antigua redacción del Código Civil francés

2. La reforma del Código Civil sobre la denominación de los animales

3. Críticas a la reforma por parte de diferentes sectores

4. El alcance de la reforma del estatuto jurídico civil de los animales

Conclusión

\section{INTRODUCCIÓN}

Como país con una tradición de derecho civil, el sistema legal francés incluye la noción de propiedad entre sus fundamentos. El animal no humano no ha escapado a esta lectura y ha sido considerado legal según el prisma de la dualidad entre algo que no pertenece a nadie y algo que le pertenece a alguien. Por lo tanto, la concepción tradicional del animal bajo la ley civil era otorgar indirectamente al animal un régimen de protección por el valor de mercado que el animal representaba para su dueño. Impulsada por los avances científicos, la ética y la evolución de las prácticas humanas, esta concepción tradicional ha sido desafiada constantemente por una verdadera reflexión social sobre el lugar de los animales en la sociedad moderna. Mientras que otros esfuerzos han tenido éxito en la modernización de la ley sobre este tema, el derecho civil francés codificado conservó una interpretación tradicional que condujo a una reforma del estatuto civil del animal en 2015.

\section{LA ANTIGUA REDACCIÓN DEL CÓDIGO CIVIL FRANCÉS}

En Francia, antes de la reforma de 2015 (Ley n 2015-177 de 16 de febrero de 2015ํ), los animales eran considerados todavía por el Código Civil como "bienes muebles" (artículo 528) o "inmuebles por destino" cuando eran colocados por el propietario de un fundo para el servicio y funcionamiento del mismo (artículo 524).

En su situación en el Código Civil, estos dos artículos se colocaron en el Libro II (De los bienes y de las diferentes modificaciones de la propiedad) por debajo del Título I (De la distinción de los bienes).

El artículo $524^{2}$ formaba parte del Capítulo I (De los inmuebles) y consideraba que "los animales y los objetos que el propietario de un terreno haya colocado allí para el servicio y la explotación de este fundo, son inmuebles por destino”. El artículo se refería específicamente a ciertas especies (animales incorporados al cultivo, palomas de las palomares, colmenas, conejos de las conejeras, los peces del agua que no se refiere al artículo 402 del Código rural y estanques referidos a los artículos 432 y 433 del mismo código) que consideraba inmuebles por destino cuando se colocaban por el propietario para el servicio y funcionamiento del fundo.

\footnotetext{
${ }^{1}$ Loi n²015-177 du 16 février 2015 : https://www.legifrance.gouv.fr/affichTexte.do?cidTexte=JORFTEXT000030248562\&categorieLien=i $\mathrm{d}$

${ }^{2}$ Article 524, Code civil (Version en vigueur du 14 mai 2009 au 18 février 2015) : https://www.legifrance.gouv.fr/affichCodeArticle.do;jsessionid=B819DF09F10706FF914EDD4E0965 92F7.tpdila15v 2?idArticle=LEGIARTI000020616199\&cidTexte=LEGITEXT000006070721\&catego $\underline{\text { rieLien}=i d \& d a t e T e x t e}=20150217$
} 
El artículo $528^{3}$ formaba parte del Capítulo II (De los muebles) y declaraba que "son muebles por su naturaleza los animales y los cuerpos que pueden transportarse de un lugar a otro, bien porque se muevan por sí mismos o bien porque sólo puedan cambiar de lugar por el efecto de una fuerza externa."

Además de estos dos artículos principales, el artículo $533^{4}$ (igualmente en el Capítulo II De los muebles) afirmaba que "la palabra 'muebles', utilizada en las disposiciones de la ley o del hombre, sin más adición o designación, no incluye el dinero en efectivo, las joyas, las deudas activas, los libros, las medallas, los instrumentos de la ciencia, los artes y la artesanía, la ropa de cuerpo, los caballos, los carruajes, las armas, los granos, el vino, el heno y los otros productos básicos; Tampoco incluye lo que es objeto de comercio.”

El estatuto jurídico civil de los animales antes de la reforma venía establecido principalmente por los artículos 524 y 528, y difería en gran medida de las disposiciones del Código Rural y el Código Penal que ya reconocían, respectivamente, explícita e implícitamente a los animales como seres vivos y sensibles.

Desde 1976, el artículo L 214-1 del Código Rural ${ }^{5}$ considera explícitamente al animal como un ser sensible a través de las disposiciones del artículo 9 de la ley $\mathrm{n}^{\circ}$ 76-629 de 10 de julio de 1976, relativa a la protección de la naturaleza ${ }^{6}$, que establece que:

"todo animal siendo un ser sensible debe ser colocado por su propietario en condiciones compatibles con los imperativos biológicos de su especie”.

En cuanto a las disposiciones penales, estas han sido objeto de una evolución extensa $^{7}$ desde 1850 y la Ley Grammont ${ }^{8}$, y se establecen en el actual artículo 521-1 del Código Penal ${ }^{9}$ francés. Este artículo castiga "la imposición, en público o de otra manera, de maltrato grave, incluyendo el maltrato sexual, o la comisión de un acto de crueldad sobre cualquier animal doméstico o amansado, o cualquier animal mantenido en cautividad, se castiga con un encarcelamiento de dos años y una multa de 30.000 euros.” Relativo al abandono, "se castiga con las mismas penas también el abandono de un animal de compañía, domesticados o en cautividad, con la excepción de los animales para la repoblación”.

El estatuto penal de los animales se considera a menudo implícito debido a que, durante la reforma del Código Penal de 1994, la mayor parte de las infracciones contra los animales fueron colocadas fuera de la categoría de delitos contra la propiedad. Las infracciones contra los animales se incluyeron en el Libro V del Código Penal "Otros

\footnotetext{
${ }^{3}$ Article 528, Code civil (Version en vigueur du 7 janvier 1999 au 18 février 2015) :

https://www.legifrance.gouv.fr/affichCodeArticle.do;jsessionid=B819DF09F10706FF914EDD4E0965 92F7.tpdila15v_2?idArticle=LEGIARTI000006428711\&cidTexte=LEGITEXT000006070721\&catego rieLien $=$ id\&dateTexte $=20150217$

${ }^{4}$ Article 533, Code civil (Version en vigueur du 4 février 1804 au 18 février 2015) : https://www.legifrance.gouv.fr/affichCodeArticle.do;jsessionid=B819DF09F10706FF914EDD4E0965 92F7.tpdila15v_2?idArticle=LEGIARTI000006428742\&cidTexte=LEGITEXT000006070721\&catego rieLien $=$ id\&dateTexte $=20150217$

${ }^{5}$ Article L214-1, Code rural :

https://www.legifrance.gouv.fr/affichCodeArticle.do;jsessionid=B819DF09F10706FF914EDD4E0965 92F7.tpdila15v_2?idArticle=LEGIARTI000022200245\&cidTexte=LEGITEXT000006071367\&catego rieLien=id\&dateTexte $=$

${ }^{6}$ Loi n ${ }^{\circ} 76-629$ du 10 juillet 1976 relative à la protection de la nature :

http://www.ineris.fr/aida/consultation document/2195

${ }^{7}$ Para más información ver: http://revistes.uab.cat/da/article/view/v5-n1-laimene/112

${ }^{8}$ Loi du 2 juillet 1850 dite Grammont sur les mauvais traitements envers les animaux domestiques : https://www.legifrance.gouv.fr/affichTexte.do?cidTexte=JORFTEXT000000332380\&fastPos=1\&cate gorieLien=id\&oldAction=rechTexte

${ }^{9}$ Article 521-1, Code pénal :

https://www.legifrance.gouv.fr/affichCodeArticle.do?idArticle=LEGIARTI000006418952\&cidTexte= LEGITEXT000006070719
} 
crímenes y delitos” y no en el Libro III “Crímenes y delitos contra la propiedad”. El artículo 521-1 está colocado en el Capítulo individual "Maltrato grave o crueldad hacia los animales” dentro del Libro V "Otros crímenes y delitos".

La flagrante incoherencia entre las disposiciones del Código Civil antes de la reforma, el Código Penal y el Código rural, unido a la demanda social de reconocer el lugar de los animales en la sociedad, desembocó en la reforma del Código Civil en 2014-2015.

\section{LA REFORMA DEL CÓDIGO CIVIL SOBRE LA DENOMINACIÓN DE LOS ANIMALES}

La reforma del estatuto civil de los animales se realizó a través del Proyecto de Ley de modernización y simplificación del Derecho en los ámbitos de la Justicia y Asuntos de Interior (No. 1808). La enmienda $n^{\circ} 59^{10}$ que contiene esta reforma fue introducida el 11 de abril de 2014 en la Asamblea Nacional (Cámara baja del Parlamento francés) por los diputados Sr. Glavany, Sra. Capdevielle, Sra. Untermaier y los miembros del Grupo Socialista, Republicano y Ciudadano.

La enmienda $n^{\circ} 59$ propuso la modificación del Código Civil, introduciendo un artículo 515-14 antes del Título I (De la distinción de los bienes) en el Libro II (De los bienes y de las diferentes modificaciones de la propiedad). El propuesto artículo 515-14 decía lo siguiente:

"Los animales son seres vivos dotados de sensibilidad. Bajo reserva de leyes que los protegen, los animales están sujetos al régimen de los bienes tangibles”.

La reforma final mantendrá el artículo escrito como tal, pero suprime el término "tangibles".

El artículo 522 se modificó para reflejar que los animales que el propietario de la tierra deja al agricultor para su cultivo no deben ser considerados inmuebles por destino, sino animales sometidos a las normas de los bienes inmuebles por destino (primer párrafo). Del mismo modo, los animales que el propietario de la tierra da como ganado a otras personas no se deben considerar como bienes muebles, sino como animales sometidos al régimen de los bienes muebles (segundo párrafo).

La enmienda propuso modificar la redacción del artículo 524 para sustituir las palabras “animales y los objetos” por "los bienes” (primer párrafo) y eliminar las referencias a los animales incorporados al cultivo, palomas de las palomeras, conejos de las conejeras, los peces del agua que no se refiere al artículo 402 del Código Rural, y a estanques referidos a los artículos 432 y 433 del mismo Código (los párrafos tercero, sexto, séptimo y noveno se eliminaron). La reforma definitiva sólo incorporaba una parte de los cambios propuestos por la enmienda y establece la siguiente nueva redacción:

"Los animales que el propietario de un terreno colocó en él, con el mismo propósito están sometidos al régimen de los inmuebles por destino".

Se propuso una nueva redacción del artículo 528 con el fin de eliminar la referencia a los animales. Según el actual artículo 528 "son muebles por su naturaleza los bienes que se pueden transportar de un lugar a otro". La palabra "caballos” se eliminó del artículo 533.

El artículo 564 también se modificó para reflejar que las palomas, los conejos y los peces en este artículo no son cosas.

Se propuso también la derogación del artículo 2.501 que consideraba como bienes inmuebles por destino a los peces de los estanques que no tienen comunicación con ríos, canales y arroyos, a los peces criados y a las jaulas de peces. Sin embargo, el texto final

\footnotetext{
${ }^{10}$ Amendement n59 présenté par M. Glavany, Mme Capdevielle, Mme Untermaier et les membres du groupe socialiste, républicain et citoyen : http://www.assembleenationale.fr/14/amendements/1808/AN/59.asp
} 
aprobado no deroga el artículo, sino que modifica su contenido para decir que estos peces están sometidos al régimen de los bienes inmuebles por destino (como en el artículo 522).

En resumen, según sus autores la reforma del Código Civil tuvo la intención de reconocer al animal como ser vivo para conciliar mejor su clasificación jurídica y su valor emocional y al mismo tiempo para lograr un régimen jurídico coherente sobre los animales, con el fin de armonizar los diferentes Códigos franceses. Esta reforma supuso la modernización de la ley y la posibilidad de ofrecer una definición legal del animal, como ser vivo y sensible, sometiendo expresamente a los animales al régimen jurídico de los bienes tangibles con una remisión expresa a las leyes especiales que los protegen.

\section{CRÍTICAS A LA REFORMA POR PARTE DE DIFERENTES SECTORES}

\subsection{Una enmienda poco progresista para los defensores de los animales}

La enmienda $\mathrm{n}^{\circ} 59$ ha sido objeto de fuertes críticas por parte de los defensores de los animales. Por ejemplo, la diputada Sra. Laurence Abeille consideró que la enmienda no era tan ambiciosa ${ }^{11}$ como el Proyecto de Ley propuesto por el Grupo de trabajo sobre la "Protección de los Animales" de la Asamblea Nacional. Este Proyecto de Ley era considerado más completo y eficaz para dar lugar a una reforma más ambiciosa de la situación jurídica del animal. Como muchos, Laurence Abeille consideró que la enmienda n59 estaba operando una reforma cosmética que en realidad no cambiaba nuestra relación con los animales, ya sean animales domésticos, animales salvajes o animales de granja.

La reforma deseada por la diputada Abeille hubiera logrado un verdadero respeto a las necesidades biológicas de los animales. En su opinión, en lugar de modificar algunos artículos del Código Civil, su reforma habría sido más ambiciosa y crítica con las prácticas que no respetan el carácter sensible de los animales.

\subsection{Una enmienda demasiado progresista para la Federación Nacional de Asociaciones de Agricultores (FNSEA)}

La enmienda $n^{\circ} 59$ también fue fuertemente criticada por la Federación Nacional de Sindicatos de Agricultores (FNSEA), que lamentaron la decisión de los parlamentarios de reconocer que los animales están 'dotados de sensibilidad' por temor a que este cambio en el Código Civil “cuestionase la práctica de la agricultura”. En una carta abierta ${ }^{12}$ al presidente Hollande, el presidente de la FNSEA dijo que este desarrollo legal habría podido cuestionar la práctica de la agricultura, debilitar la propia experiencia práctica de los ganaderos relativa al bienestar animal y ofrecer una oportunidad para el movimiento a favor de los derechos de los animales para aumentar los procedimientos judiciales contra los ganaderos.

Desde el punto de vista puramente jurídico, los temores expresados por la FNSEA, incluyendo el cuestionamiento de la ganadería, no parecen basarse en argumentos legales puesto que los animales siguen sometidos al régimen de los bienes según el artículo 515-14 del Código Civil y, además, el Código Rural, primer texto de aplicación para los agricultores, ya reconocía la condición de los animales como seres sensibles desde 1976 (artículo L 2141 o artículo 9 ley n ${ }^{\circ} 76-629$ de 10 de julio de 1976 relativa a la protección de la naturaleza). Por lo tanto, la oposición expresada por la FNSEA parece basarse más en consideraciones políticas que jurídicas.

\subsection{La reforma final llevada a cabo por la Ley $n^{\circ}$ 2015-177 del 16 de febrero 2015}

\footnotetext{
${ }^{11}$ Assemblée nationale, XIVe législature, Session ordinaire de 2013-2014, Compte rendu intégral, Troisième séance du mardi 15 avril 2014 : http://www.assemblee-nationale.fr/14/cri/20132014/20140190.asp

${ }^{12}$ http://www.revenuagricole.fr/images/pdf/lettre-xavier-beulin-francois-hollande.pdf

76 Derecho Animal. Forum of Animal Law Studies, vol. 9/3
} 
La Ley n 2015-177, de 16 de febrero 2015, modificó el Código Civil para reformar el estatuto jurídico civil de los animales y, tal y como se ha señalado anteriormente, se hicieron muy pocos cambios a la redacción propuesta por la enmienda $\mathrm{n}^{\circ} 59$. establece que:

Sin lugar a dudas, el artículo $515-14^{13}$ es el artículo principal de esta reforma y

"Los animales son seres vivos dotados de sensibilidad. Bajo reserva de leyes que los protegen, los animales están sujetos al régimen de los bienes”.

El artículo 515-14, simbólicamente, se incluye en el Libro II: De los bienes y de las diferentes modificaciones de la propiedad, pero antes del Título I: De la distinción de los bienes.

\section{EL ALCANCE DE LA REFORMA DEL ESTATUTO JURÍDICO CIVIL DE LOS ANIMALES}

La redacción de este nuevo artículo parece, como en el caso del artículo 511-1, 3) del Código Civil catalán ${ }^{14}$, tener un alcance simbólico porque, aunque considera a los animales como seres vivos dotados de sensibilidad, el régimen jurídico de la propiedad sigue siendo aplicable a los animales.

\section{1. ¿Hacia una interpretación progresista de los jueces?}

A pesar de lo anterior, como señaló el eminente jurista en Derecho Animal francés, el Prof. Jean-Pierre Marguénaud, el alcance de esta reforma, aun cuando pueda tildarse de simbólico, es importante ${ }^{15}$ porque, aunque no cambia la condición animal drásticamente, la interpretación de los jueces podría ser influenciada en gran parte por esta reforma del Código Civil gracias a la armonización de la consideración de los animales en los códigos franceses. Como se mencionó anteriormente, el animal es un ser "sensible” en el artículo L-214-1 del Código Rural mientras el Código Penal castiga en su artículo 521-1 la imposición, en público o de otra manera, de maltrato grave, incluyendo el maltrato sexual, o la comisión de un acto de crueldad sobre cualquier animal doméstico o amansado, o cualquier animal mantenido en cautividad, así como el abandono de un animal. Ahora el Código Civil valida ese estatuto de ser vivo dotado de sensibilidad. Esta armonización apoya la idea de que los animales son considerados como seres sensibles en todo el Corpus Jurídico francés que se aplica a toda la sociedad.

El profesor Marguénaud también recuerda que el Código Civil es percibido por los expertos como "el Código por excelencia" ${ }^{16}$. Por lo tanto, una categorización negativa de los animales o poco acorde con la realidad científica (antiguos artículos del Código Civil) sin duda afecta negativamente en la práctica y especialmente en la interpretación judicial en relación con los animales, aún más en los casos relativos a los artículos del Código Rural o del Código Penal.

El profundo desfase entre las disposiciones del Código Penal y del Código Rural (que garantizaban hasta ahora una protección mínima a los animales) y la realidad judicial (multas

\footnotetext{
${ }^{13}$ Article 515-14, Code Civil (Version en vigueur au 18 février 2015) : https://www.legifrance.gouv.fr/affichCodeArticle.do?idArticle=LEGIARTI000030250342\&cidTexte= LEGITEXT000006070721

${ }^{14}$ Artículo 511-1, 3), Código Civil de Cataluña: http://civil.udg.edu/normacivil/cat/CCC/ES/L52006.htm

${ }^{15}$ http://www.lepopulaire.fr/limoges/vie-pratique-consommation/2014/04/22/serie-animaux-un-statutjuridique-ambigu-pour-le-juriste-limougeaud-jean-pierre-marguenaud 1975976.html

${ }^{16}$ Carbonnier J., « Les lieux de mémoire », tome II, vol. 2, direction P. Nora (Gallimard 1986) p. 293

Derecho Animal. Forum of Animal Law Studies, vol. 9/3
} 
ínfimas y pocas penas de prisión) podía hasta antes de la reforma del Código Civil explicarse por el hecho de que este Código representa la referencia definitiva para los jueces franceses, y ello a pesar de que el Código Penal ya castigaba el maltrato animal grave y la crueldad hacia los animales con una multa máxima de 30.000 euros y hasta dos años de prisión.

Con la nueva redacción del Código Civil, es posible esperar que la armonización de una consideración en todo el Derecho francés de los animales como seres sensibles se traduzca en una mejora en la interpretación legal de los jueces en los casos referidos a los animales, tanto en materia civil como penal y, especialmente en el ámbito penal, en los casos de maltrato o crueldad hacia los animales.

\subsection{Las corridas de toros y peleas de gallos permanecen legales}

Aunque el cambio del estatuto jurídico civil operado permite una interpretación más progresista por los jueces en materia civil y penal, este cambio no tendrá ningún impacto sobre las disposiciones legales relativas al maltrato animal en virtud del Código Penal ni sobre la tauromaquia u otras actividades consideradas legales que tienen relación con los animales.

De hecho, la tauromaquia se volvió una práctica legal en Francia en 1951. Un párrafo relativo a la tauromaquia o "corridas de toros", como menciona la ley, fue introducido en el artículo único de la Ley Grammont. La Ley n51-461 de 24 de abril de 1951, complementando la Ley del 2 de julio de 1850 sobre el maltrato hacia los animales domésticos ${ }^{17}$, convirtió la tauromaquia en una práctica legal. Tal y como fue redactado el texto de la citada ley, "las previsiones del presente artículo no son aplicables a las corridas de toros donde se puede demostrar una tradición ininterrumpida. En 1959, el Decreto n591051 reprimiendo el maltrato hacia los animales ${ }^{18}$ añadió el adjetivo "local”.

Respecto a las peleas de gallos, la Ley $n^{\circ} 64-690^{19}$ introdujo, después del párrafo relativo a las corridas de toros, la frase siguiente:

"Tampoco ellas [las previsiones contra el maltrato] se aplican a las peleas de gallos en localidades donde una tradición ininterrumpida puede ser establecida”.

Queda claro, pues, que la reforma del estatuto jurídico civil de los animales operada a través del Código Civil francés y que reconoce que todos los animales son seres vivos sensibles, no tiene ninguna trascendencia legal ni para las corridas de toros, ni para las peleas de gallos, ni para todas aquellas otras actividades (caza, pesca, ganadería, etc.) relacionadas con los animales que según el Ordenamiento Jurídico son prácticas legales.

\section{CONCLUSIÓN}

Si bien es innegable que la reforma del estatuto civil de los animales en Francia cumple el objetivo de armonizar la consideración de los animales por la ley, por una parte, con respecto a las otras disposiciones legales en vigor y, por otra, con respecto a la evolución de la sociedad, el impacto de esta reforma sigue siendo incierto. Esta reforma, considerada insuficiente por algunos y amenazante por otros, reconoce el carácter sensible del animal y acentúa la incoherencia entre esta denominación y las prácticas legales que siempre tienen un efecto nocivo, directa o indirectamente, sobre el bienestar animal.

\footnotetext{
${ }^{17}$ Loi n 51-461 du 24 avril 1951 portant modification de la loi du 02-07-1850 relative aux mauvais traitements envers les animaux : https://www.legifrance.gouv.fr/affichTexte.do?cidTexte=JORFTEXT000000336230\&dateTexte=

${ }^{18}$ Décret $\mathrm{n}^{\circ}$ 59-1051 du 7 septembre 1959 reprimant les mauvais traitements exerces envers les animaux : https://www.animallaw.info/sites/default/files/stfrdecreeno59_1051.pdf

${ }^{19}$ Loi n 64-690 du 8 juillet 1964 modifiant la loi $n^{\circ}$ 63-1143 du 19 novembre 1963 relative à la protection des animaux : https://www.animallaw.info/sites/default/files/stfrlawno64_690.pdf
} 
A pesar de esta observación, esta reforma marca un paso importante no solo en la historia del sistema legal francés, sino también para un gran número de países de tradición de derecho civil que podrían ver una oportunidad para reformar su sistema legal interno con el objetivo de rehabilitar el lugar del animal en una sociedad moderna. 